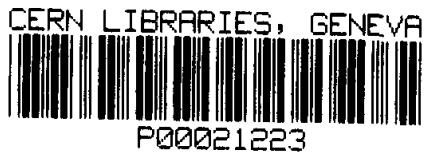

\title{
A TEST OF SPECIAL RELATIVITY WITH STORED LITHIUM IONS
}

R. Grieser, R. Klein, G. Huber, S. Dickopf, I. Klaft, P. Knobloch, P. Merz

Institut für Physik, Universität Mainz

F. Albrecht, D. Habs, D. Schalm

Max Planck Institut für Kernphysik, Heidelberg

T. Kühl

GSI, Darmstadt

\begin{abstract}
Laser spectroscopy at the heavy ion storage ring TSR in Heidelberg allows for precision experiments testing the limits of the special theory of relativity. With an optical $\Lambda$ type three level system of ${ }^{7} \mathrm{Li}^{+}$the Doppler shift has been measured by saturation spectroscopy as a test of the time dilatation factor $\gamma=\left(1-\beta^{2}\right)^{-1 / 2}$ at an ion velocity of $\mathrm{v}=6.4 \%$ c. A precision of $\Delta v / v<9.10^{-9}$ has been obtained, which sets a second order limit of $1.1 \cdot 10^{-6}$ for any deviation from the time dilatation factor. The fourth order limit of this deviation is set below $2.7 \cdot 10^{-4}$ by the present experiment. These limits are given at a $1 \sigma$ confidence level.
\end{abstract}

(ISOLDE - General)

Submitted to Appl. Phys. B 



\title{
A test of special relativity with stored Lithium ions
}

\author{
R.Grieser ${ }^{1}$, R.Klein ${ }^{1}$, G.Huber ${ }^{1}$, S.Dickopf ${ }^{1}$, I.Klaft ${ }^{1}$, P.Knobloch ${ }^{1}$ \\ P.Merz ${ }^{1}$, F.Albrecht ${ }^{2}$, M.Grieser ${ }^{2}$, D.Habs ${ }^{2}$ D.Schwalm ${ }^{2}$, T. Kühl ${ }^{3}$ \\ 1 Institut für Physik, Universität Mainz, D-55099 Mainz, Fed. Rep. Germany \\ 2 Max Planck Institut für Kernphysik Heidelberg, D-69029 Heidelberg, Fed. Rep. Germany \\ 3 Gesellschaft für Schwerionenforschung, D-64220 Darmstadt, Fed. Rep. Germany
}

\begin{abstract}
Laser spectroscopy at the heavy ion storage ring TSR in Heidelberg allows for precision experiments testing the limits of the special theory of relativity. With an optical $\Lambda$ type three level system of ${ }^{7} L i^{+}$the Doppler shift has been measured by saturation spectroscopy as a test of the time dilatation factor $\gamma=\left(1-\beta^{2}\right)^{-1 / 2}$ at an ion velocity of $\mathrm{v}=6.4 \% \mathrm{c}$. A precision of $\frac{\Delta \nu}{\nu}<9 \cdot 10^{-9}$ has been obtained, which sets a second order limit of $1.1 \cdot 10^{-6}$ for any deviation from the time dilatation factor. The fourth order limit of this deviation is set below $2.7 \cdot 10^{-4}$ by the present experiment. These limits are given at a $1 \sigma$ confidence level.
\end{abstract}

\section{Introduction}

Since its formulation at the beginning of this century the special theory of relativity has had continuous impact on physics and is now ever present up to many body descriptions of solids and nuclei. The first experimental support was found in the Michelson Morley experiment showing an isotropic speed of light in a symmetric interferometer roundtrip [1]. Later on Kennedy and Thorndike showed the isotropy of the length in the null result of a similar experiment with an unsymmetrical two arm interferometer [2]. These key experiments have their modern counterparts in laser interferometric tests which set upper limits for the spatial asymmetry to $5 \cdot 10^{-9}$ and $7 \cdot 10^{-5}$ [3]. Local Lorentz invariance of spin and mass dependent interactions has been reported [4].

Beyond these invariance tests of special relativity, the validity of the transverse Doppler shift was first tested by Ives and Stilwell [5]. The corresponding factor $\gamma=\left(1-\beta^{2}\right)^{-1 / 2}$ governing time dilatation, length contraction, relativistic mass increase and related effects 
is apparent in all fields of physics and has been tested meanwhile directly or implicitly in numerous experiments. These tests may be compared with respect to the velocity of the test particle relative to the observer's laboratory, though most of the low velocity experiments are considering a preferential reference frame comoving at $350 \mathrm{kms}^{-1}$ with the universe mass center as seen from the dipole asymmetry of the $3 \mathrm{~K}$ background radiation [7]. At high velocities these tests of $\gamma$ are mostly independent of this cosmic motion.

The significance of tests in special relativity can be described in terms of test theories, allowing for small deviations from the Lorentz transformation, but conserving the basic transformation properties. Most of the analysis refers to the initial work of Mansouri and Sexl [6] and a modern analysis of Ives Stilwell type experiments is given by [8].

In the present experiment Lithium ions are used as moving clocks with optical frequencies $\nu_{1,2}$ at rest, moving in a well controlled trajectory in the TSR storage ring at a velocity $\beta=\frac{v}{c}$ relative to the laboratory. The Doppler shifted frequencies are given by

$$
\nu=\nu_{1,2}(\gamma(1+\beta \cos (\theta)))^{-1}
$$

with the laboratory angle $\theta$ between ion beam and direction of observation. In a collinear saturation spectroscopy with parallel and antiparallel laser beams of frequency $\nu_{p}$ and $\nu_{a}$ respectively, the relation $\nu_{a} \cdot \nu_{p}=\nu_{1} \cdot \nu_{2}$ is exact within the framework of special relativity since $\gamma^{2}\left(1-\beta^{2}\right)=1$.

Any deviation given as $\gamma=\gamma_{S R T}\left(1+\delta \alpha \beta^{2}+\delta \alpha_{2} \beta^{4}+\ldots\right)$ can be tested to second order by

$$
\nu_{a}=\frac{\nu_{1} \nu_{2}}{\nu_{p}}\left(1+2 \delta \alpha\left(\beta^{2}+2 \beta \beta^{\prime} \cos (\Omega)\right)\right)
$$

where $\beta^{\prime}$ is the velocity of the above mentioned motion relative to the $3 \mathrm{~K}$ background and $\Omega$ the angle between ion motion and the cosmic motion [9]. The term $\beta^{2}$ is sensitive on absolute deviations and the term $\beta \beta^{\prime} \cos (\Omega)$ on sidereal modulation of deviations from special relativity.

Several laser experiments have been reported and analyzed for limits on $\delta \alpha$. Three of them have been performed as two photon spectroscopy on a fast Neon atomic beam at $v=0.36 \% c$ [11] [12], [13] resulting in a precision of $2.3 \cdot 10^{-6}$ [12] with a corresponding $\delta \alpha<1.6 \cdot 10^{-6} 1$ for an absolute limit and $\delta \alpha<1.4 \cdot 10^{-6}[13]$ for the limit on sidereal modulation. The previous version of the present experiment has resulted in $\delta \alpha \leq 1.5 \cdot 10^{-5}$

\footnotetext{
${ }^{1}$ This limit is deduced from table (1) in reference [12] using the analysis of reference [8] and [9]
} 
[14] the limit being mainly due to uncertainties in the angular control of the ion beam and in the accuracy of the frequency determination. ${ }^{2}$

The results from other test experiments including Mößbauer rotor and $\mathrm{g}-2$ experiments on stored muons and electrons are considered at the end of this paper.

\footnotetext{
${ }^{2}$ When comparing collinear saturation spectroscopy where the resonance is driven with two independent lasers and two photon spectroscopy driven with a single laser, one has to remind that the relation for testing special relativity $\frac{\nu_{s} \nu_{Y}}{\nu_{1} \nu_{2}}$ is equivalent to $\left(1-\beta^{2}\right) \gamma^{2}=1$ whereas the relation $\frac{\nu_{k}}{\sqrt{\nu_{2} \nu_{2}}}$ is equivalent to $\sqrt{1-\beta^{2}} \gamma=1$. This quadratic relation introduces a factor 2 in equation (2) as compared to $\frac{\nu_{L}}{\sqrt{\nu_{1} v_{2}}}=\left(1+\alpha \beta^{2}+\right.$ higher orders $)$ for the two photon tests. This apparent discrepancy vanishes when the limits are related to the frequency errors $\alpha \beta^{2} \leq \frac{1}{2} \sqrt{\delta \nu_{a}^{2}+\delta \nu_{p}^{2}}$ for independent and uncorrelated errors or $\alpha \beta^{2} \leq \frac{1}{2} \sqrt{\left(\left|\delta \nu_{a}\right|+\left|\delta \nu_{p}\right|\right)^{2}}$ for correlated errors. this expression is identical with $\alpha \beta^{2} \leq \delta \nu_{L}$ for the two photon experiments, where $\nu_{a}=\nu_{p}=\nu_{L}$. This aspect has not been recognized in [12] where our pervious experiment has been analyzed incorrectly on the basis of the two photon equations.
} 


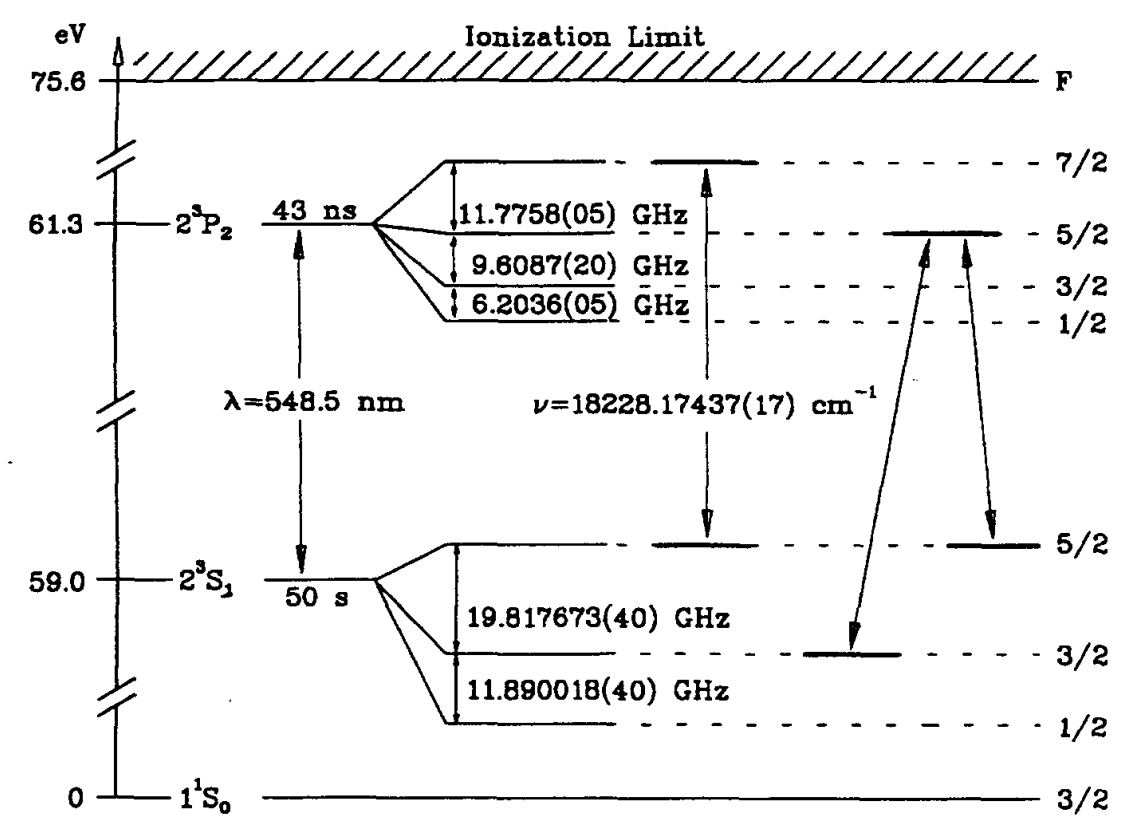

Figure 1: Spectrum of the heliumlike ${ }^{7} \mathrm{Li}^{+}$triplet system. The transitions relevant for the experiment are indicated by the arrows. The transition frequency of the $2^{3} S_{1} F=\frac{5}{2} \rightarrow$ $2^{3} P_{2} F^{\prime}=\frac{7}{2}$ has been determined by [15], whereas the hyperfine structure splitting has been measured by [16]. The $F=\frac{5}{2} \rightarrow F^{\prime}=\frac{5}{2}$ and the $F=\frac{3}{2} \rightarrow F^{\prime}=\frac{5}{2}$ transitions, which form a closed three level $\Lambda$ type system are used for saturation spectroscopy. The errors correspond to a $3 \sigma$ uncertainty.

\section{Experimental setup at the storage ring}

In the heliumlike spectrum of ${ }^{7} \mathrm{Li}^{+}$the triplet system shows a well resolved and precisely known fine and hyperfine structure multiplet. The relevant part of the spectrum is the ${ }^{3} S_{1} \rightarrow{ }^{3} P_{2}$ line at $\lambda=548,6 \mathrm{~nm}$ as shown in figure (1). A closed two level system for beam diagnostics $\left({ }^{3} S_{1} F=\frac{5}{2} \rightarrow{ }^{3} P_{2} F^{\prime}=\frac{7}{2}\right)$ is indicated and the transitions $\left({ }^{3} S_{1} F=\frac{3}{2} \rightarrow\right.$ $\left.{ }^{3} P_{2} F^{\prime}=\frac{5}{2}\right)$ and $\left({ }^{3} S_{1} F=\frac{5}{2} \rightarrow{ }^{3} P_{2} F^{\prime}=\frac{5}{2}\right)$ are forming a $\Lambda$ system for precision spectroscopy.

At the Max Planck-Institut für Kernphysik in Heidelberg the ion beam is first produced as a $\mathrm{Li}^{-}$and fed to the MP tandem. At the high voltage terminal singly charged ${ }^{7} \mathrm{Li}^{+}$ ions are formed by stripping in gas collisions. These ions, which are up to $20 \%$ excited to the metastable ${ }^{3} S_{1}$ state, are injected by a multiturn technique to the TSR [17]. Generally $10^{6}$ ions can be stored in the storage ring consisting of four straight sections, connected by four pairs of dipole magnets. Twenty quadrupole magnets are used for horizontal and 
vertical strong focussing.

Depending on the rest gas collisions this beam can be stored with a 10-40 sec lifetime. Its energy has been chosen close to maximum magnetic rigidity of $1.4 \mathrm{Tm}$ in order to tune the Doppler shifted ${ }^{3} S_{1} \rightarrow{ }^{3} P_{2}$ line in resonance with a co-propagating $\mathrm{Ar}^{+}$laser beam at $\lambda=514.5 \mathrm{~nm}$. This resonance occurs at $\beta=0.064$ and thus an antiparallel dye laser beam is resonant at $\lambda=584.8 \mathrm{~nm}$.

After injection the beam diameter exceeds $1 \mathrm{~cm}$ as a result of the transverse betatron motion. The Coulomb interaction with a comoving electron beam of the electron cooler [18] in the second section of the TSR reduces the transverse momentum within $6-8 \mathrm{sec}$ and thus the beam diameter to $1 \mathrm{~mm}$. Simultaneously the mean ion velocity is synchronized to the electron velocity with a longitudinal momentum spread of $\frac{\Delta p}{p}=3 \cdot 10^{-5}$. This momentum spread may be further reduced by laser cooling by almost two orders of magnitude [19], however the broader electron cooled ion beam has been chosen for the present experiment in order to minimize light shift perturbations.

In the third, experimental section of the TSR four position pick ups have been mounted at a distance of $2,4 \mathrm{~m}[20]$ and allow to measure horizontal and vertical beam position. These pick ups were aligned relative to an axis defined by two geodetic targets outside the storage ring. The alignment accuracy of the pick ups corresponds to an angular uncertainty of $\Delta \theta= \pm 240 \mu \mathrm{rad}$.

Laser beams could be sent through the experimental section via quartz windows and adjusted parallel to the ion beam. The optical resonances were observed as fluorescence at right angle by two photomultipliers.

\section{The laser frequency control}

A single mode $\mathrm{Ar}^{+}$laser stabilized on a ${ }^{127} I_{2}$ line, which is a recommended frequency standard [21], and a Coherent 699/21 ring dye laser were used for saturation spectroscopy in the storage ring. The $\mathrm{Ar}^{+}$laser line width was improved by locking the laser cavity cavity via a piezo driven mirror to a high finesse Fabry Perot interferometer. With an additional modulation voltage the laser was frequency modulated at $29 \mathrm{kHz}$ with a corresponding modulation amplitude of $2 \mathrm{MHz}$. The $P(13) 43-0 a_{3}$ hyperfine structure line in ${ }^{127} I_{2}$ was recorded by saturation spectroscopy in an external cell. The first derivative of the saturation signal was observed by phase synchronous detection and fed to the interferometer for the long term stabilization of the laser. 


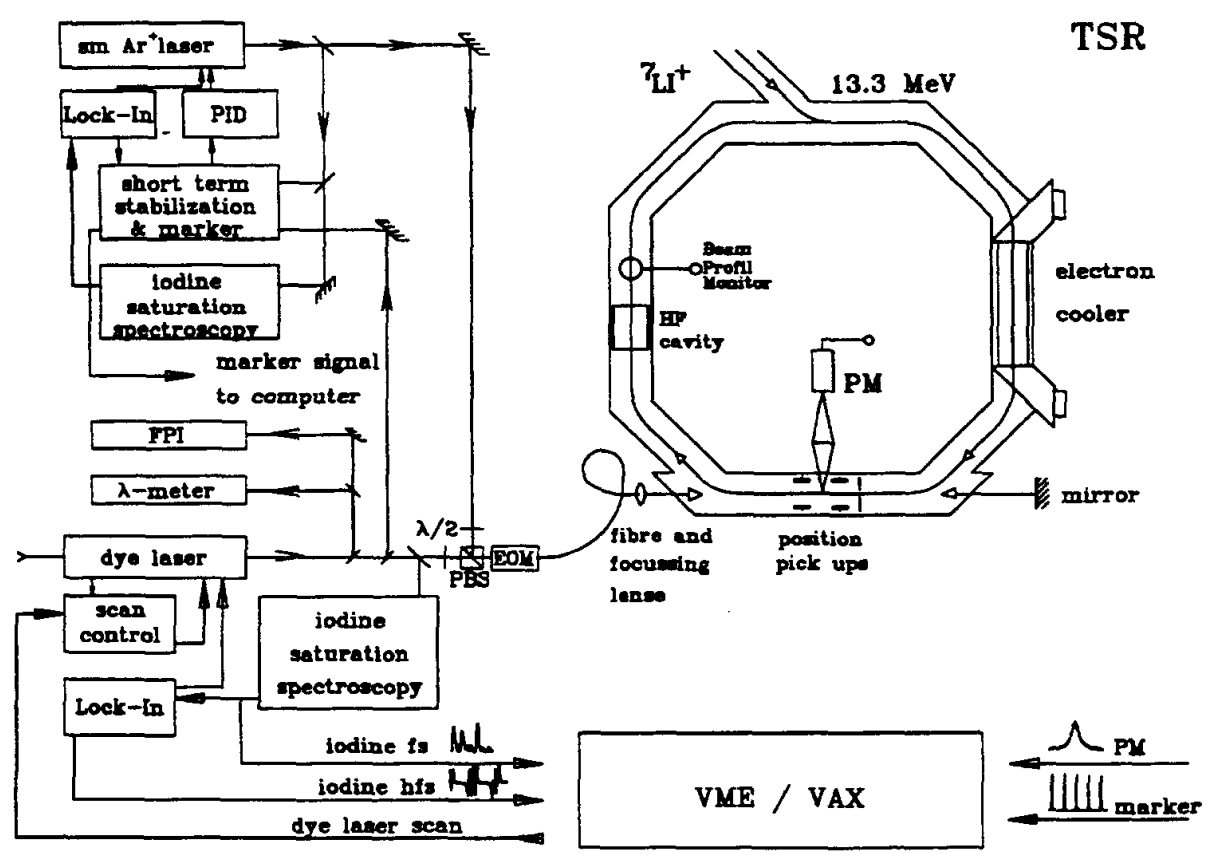

Figure 2: Schematic set up for the TSR test experiment. On the right the storage ring is shown, having a circumference of $55.6 \mathrm{~m}$. On the left a schematic of the laser frequency measurement is shown. The laser beams are sent through a single mode polarization preserving fibre to the TSR over a distance of $20 \mathrm{~m}$. The laser experiment is controlled by a VME bus computer, coupled via the ethernet link to a VAX cluster. For more details see text. 
The dye laser is a commercial laser with some modifications. The dye circulation has been replaced by a high pressure pump, a temperature stabilized cooler at $8^{\circ} \mathrm{C}$ and a sapphire jet nozzle. The resonator has been reinforced by two lateral invar rods. The laser line width was measured in the beat signal with a Helium Neon laser and a line width of $1 \mathrm{MHz}$ in the $10 \mathrm{msec}$ average of a RF spectrum analyzer has been observed. Frequency modulation of the laser has been achieved by adding the modulation voltage to the driver circuit of the piezo driven mirror at a modulation frequency of $40 \mathrm{kHz}$ and a corresponding modulation amplitude of $2.5 \mathrm{MHz}$.

The frequency of the scanning laser was determined by the simultaneous recording of ${ }^{127} I_{2}$ HFS resonances of the $R(99) 15-1$ transition and the transmission signals from the Fabry Perot interferometer serving as frequency reference for the $\mathrm{Ar}^{+}$laser and an interpolation relative to the $i$ - component of the $R(99) 15-1$ line [22].

The free spectral range of the cavity has been determined to $\Delta \nu=606(1.9) \mathrm{MHz}$ by comparing the transmission peaks of the $\mathrm{TEM}_{00}$ aligned interferometer with the simultaneously detected HFS-splitting of ${ }^{127} I_{2}$ at $543 \mathrm{~nm}$ [23]. The error for one free spectral range is due to nonlinearities in the scan operation of the laser.

The reference cavity for the $\mathrm{Ar}^{+}$laser and the saturation spectroscopy optics have been mounted on transportable steel bases in acoustically isolated boxes, in order to reduce influences of temperature changes and of the high noise level inherent in an accelerator laboratory.

The accuracy of our frequency resolution is given by the uncertainties of the known ${ }^{127} I_{2}$ reference lines and the uncertainties of the laser frequency measurements itself. During the signal scanning the frequency measurement was limited by the coarse digital registration of the saturation signals to $\Delta \nu= \pm 0.85 \mathrm{MHz}$.

\section{Experiment and data taking}

The experimental set up as described in the preceeding sections is shown in figure (2). The laser beams with parallel polarization were overlapped and sent through a polarization preserving single mode fibre over a distance of $20 \mathrm{~m}$ to the entrance window of the experimental section of the TSR. The laser beams emerging from the fibre end entered a beam expander consisting a microscope objective $(\times 10)$ and an achromat $(f=600 \mathrm{~mm})$, mounted on a step motorized $x y-\theta \phi$ positioning stage. The bichromatic beam at the beam expander exit showed a $11.6 \mathrm{~mm} \frac{1}{e}$ diameter and was focussed on a plane back- 


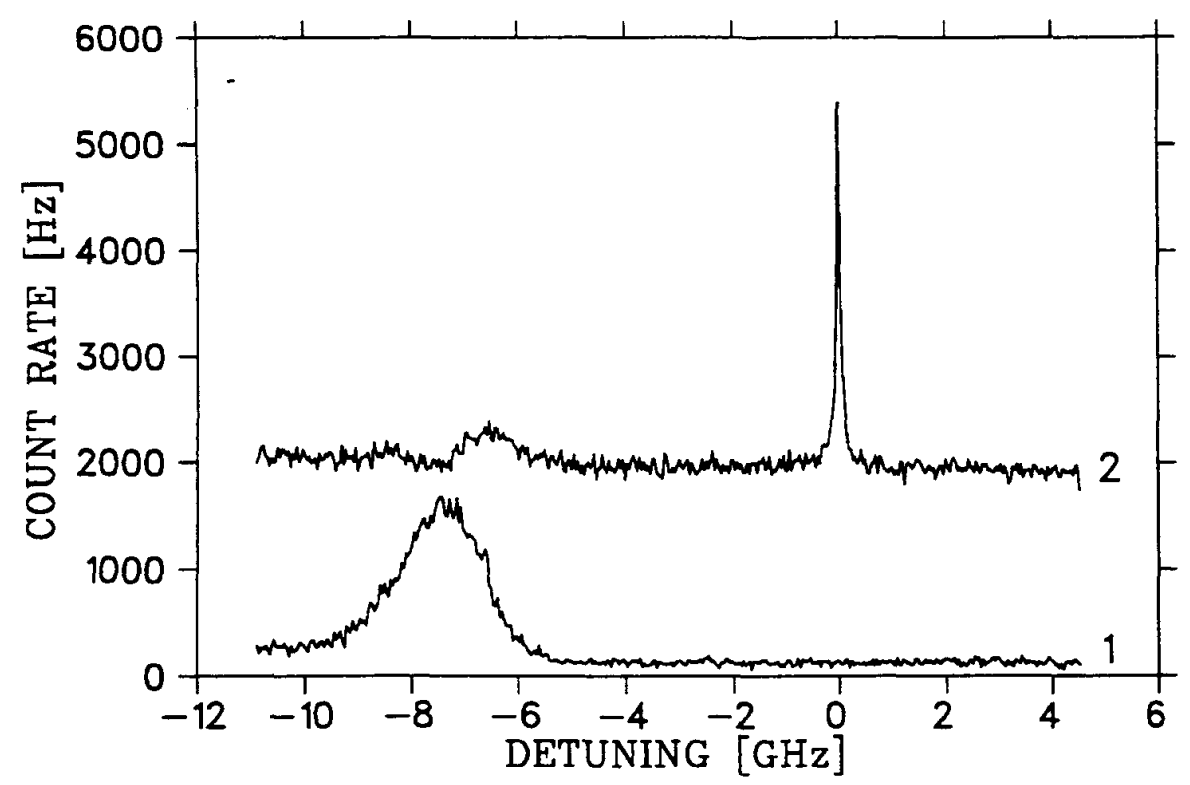

Figure 3: Fluorescence signals from the electron cooled ion beam. In trace 1 only the dye laser light is sent to the experiment, and the Doppler broadened fluorescence of the $\mathrm{F}=\frac{5}{2} \rightarrow \mathrm{F}^{\prime}=\frac{7}{2}$ transition is visible. The HWHM corresponds to $\frac{\Delta v}{v}=3 \cdot 10^{-5}$. In trace 2 both lasers are sent to the ring, which increases the background due to the stray light from the $\mathrm{Ar}^{+}$laser. The $\mathrm{Ar}^{+}$laser at resonance with the $\mathrm{F}=\frac{5}{2} \rightarrow \mathrm{F}^{\prime}=\frac{5}{2}$ transition optically pumps the resonant ions into the $F=\frac{3}{2}$ level and the dye laser scan shows the almost depleted $F=\frac{5}{2}$ level at the $F=\frac{5}{2} \rightarrow F^{\prime}=\frac{7}{2}$ transition. The resonance $F=\frac{3}{2} \rightarrow F^{\prime}$ $=\frac{5}{2}$ yields a strong fluorescence from the simultaneous excitation of the $\Lambda$-system. 
reflecting mirror behind the experimental section at $15 \mathrm{~m}$ distance. The collinear laser beams were aligned to the geodetic targets, which also served for the alignment of the pick up devices, with a resulting accuracy of $2 \mathrm{~mm}$ over a distance of $14 \mathrm{~m}$.

The retro reflected laser beams were adjusted for maximum transmission through the fibre, thereby an angular accuracy of $\Delta \phi< \pm 40 \mu \mathrm{rad}$ could be achieved.

The laser powers were adjusted to an almost equal saturation of both transitions of the $\Lambda$ system, and the total power was controlled by an electro optical modulator at the fibre input. The optical resonances with the ion beam were detected as fluorescence by two photomultipliers, equipped with interference filters and colour glasses to suppress stray light. One of them was equipped with an imaging system, consisting of a condensor lens ( $f=250 \mathrm{~mm}, \emptyset=150 \mathrm{~mm}$ ) and a light guide collecting light from the beam image.

After the alignment of the ion beam to the zero position of the pick ups, spectra were taken as follows:

The injected ion beam was first electron cooled for $7 \mathrm{sec}$. During this time the laser beams were blocked mechanically in order to avoid optical pumping during the longitudinal velocity change associated with the electron cooling.

Then the dye laser was scanned across the $\Lambda$ resonance and the photon counting rates from the fluorescence, the transmission signal of the Fabry Perot interferometer, the corresponding ${ }^{127} I_{2}$ saturation signals, as well as the dye laser power and the lock signal of the $\mathrm{Ar}^{+}$laser were recorded in 400 channels each. The parameters were chosen for optimum counting rate and frequency resolution at the actual beam lifetime of $8.8 \mathrm{sec}$.Figure (4) shows a spectrum recorded by this procedure.

The experiment was controlled by a VME-bus computer who sent the data via the ethernet link directly to a VAX cluster, where the data storage and the online analysis was carried out.

After electron cooling the beam reached a transverse diameter of $1 \mathrm{~mm}$ and a longitudinal velocity distribution of $\frac{\Delta v}{v}=3 \cdot 10^{-5}$ as shown in figure (3). The mean velocity could be adjusted to match the $F=\frac{5}{2} \rightarrow F^{\prime}=\frac{5}{2}$ or $F=\frac{3}{2} \rightarrow F^{\prime}=\frac{5}{2}$ resonance with the fixed frequency $\mathrm{Ar}^{+}$laser by varying the cathode potential and thus the velocity of the electron beam of the electron cooler.

These two settings correspond to opposite residual photon recoils in the $\Lambda$ resonance . The result of these measurements are shown in figure (5), and a shift of $\Delta \nu= \pm 0.7 \mathrm{MHz}$ has been observed. 


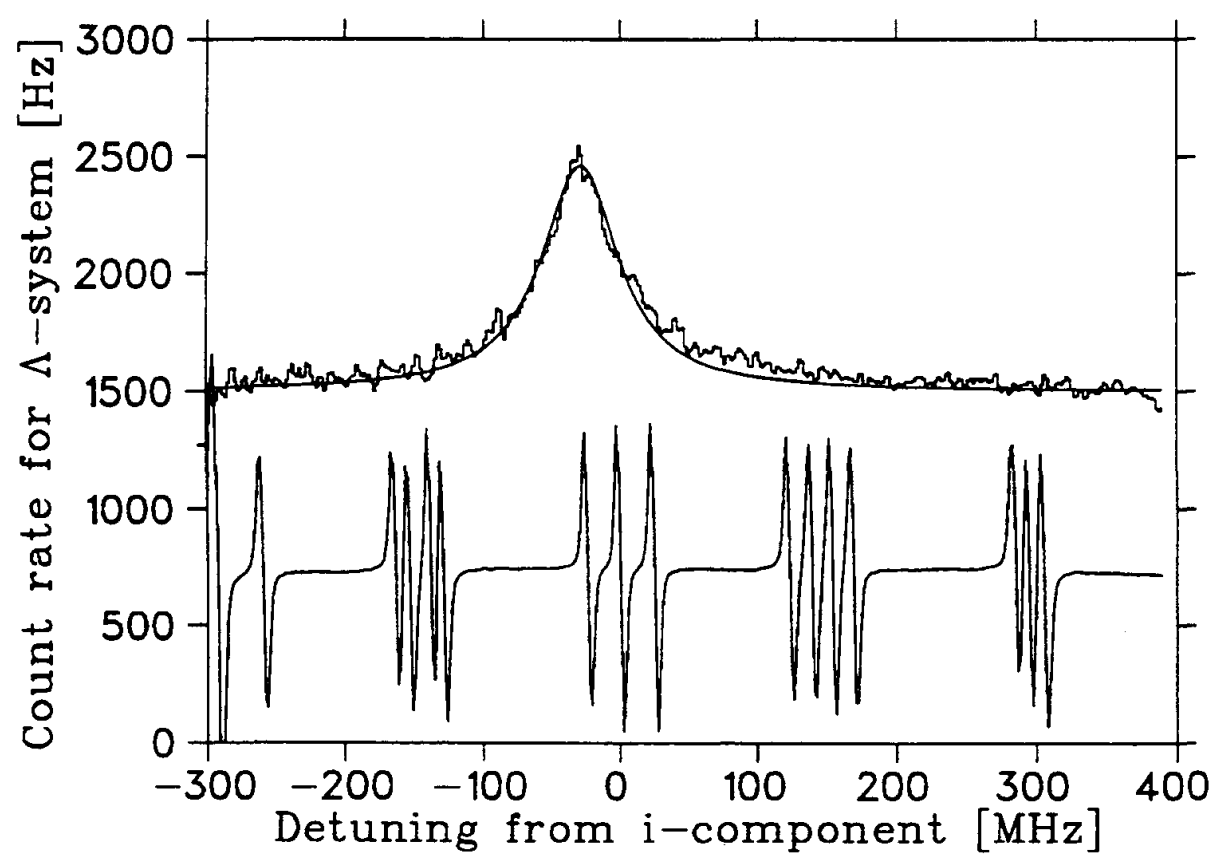

Figure 4: Fluorescence signal from a multiple scan of the $\Lambda$ resonance. The $\mathrm{Ar}^{+}$laser was locked to the $P(13) 43-0 \quad a_{3}$ component of ${ }^{127} I_{2}$ and the hyperfine structure of the $\mathrm{R}(99) 15-1$ transition in molecular Iodine is simultaneously recorded. The spectra have been corrected for the lifetime $\tau_{1 / e}=8.8 \mathrm{sec}$ of the stored ion beam and small drifts of the dye laser. In the lower trace the ${ }^{127} I_{2}$ reference lines are shown. The zero of the frequency scale corresponds to the position of the $\mathrm{R}(99) 15-1$ i-component, serving as frequency reference for the antiparallel dye laser beam.

Small shifts are induced by the AC Stark shift, when the resonance is adjusted to the wings of the velocity distribution. This shift has been measured and the results are shown in figure (6). Within the stability of the electron cooler voltage the AC Stark shift is estimated to be smaller than $\Delta \nu= \pm 1 \mathrm{MHz}$.

After a correct setup of the laser frequency, the beam alignment and the velocity adjustment for zero AC Stark shift up to 100 scans have been recorded. A total of 571 single spectra were finally analyzed for line position, amplitude and line width. Sum spectra have been generated with individual readjustment for superposition of the ${ }^{127} I_{2} R(99) 15-1$ i-component reference line. Finally the limited storage lifetime induced an exponential signal decrease during the scans, which had been corrected after subtraction of the constant background signal. This induced a frequency uncertainty of $\Delta \nu= \pm 0.5 \mathrm{MHz}$ for 


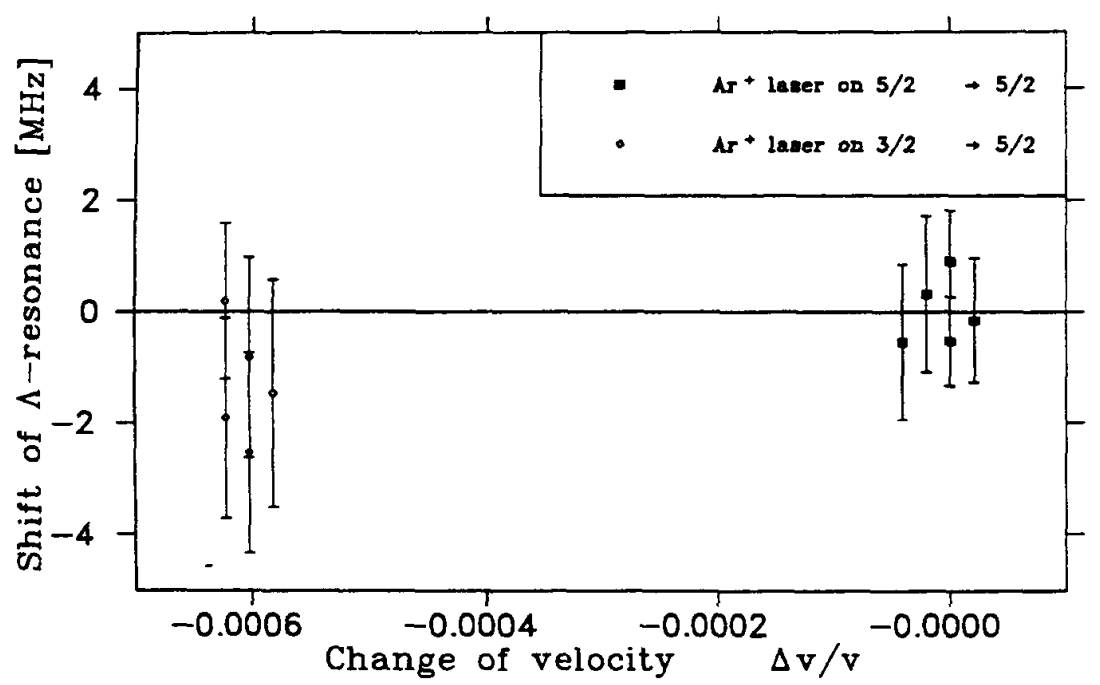

Figure 5: By varying the cathode potential of the electron cooler, the mean ion velocity is changed. The $\mathrm{Ar}^{+}$laser, initially resonant with the $\mathrm{F}=\frac{5}{2} \rightarrow \mathrm{F}^{\prime}=\frac{5}{2}$ transition is Doppler shifted to the $F=\frac{3}{2} \rightarrow F^{\prime}=\frac{5}{2}$ transition. From equation (2) follows the observed opposite shift for the dye laser's Doppler shifted frequency. The frequency shift of the $\Lambda$ resonance is due to residual velocity changes by photon recoil.

formula by $\frac{\Delta \nu}{\nu}=\frac{\phi^{2}}{2} \beta$, where $\phi$ is the angle between the lasers aligned parallel to the ion beam and $\frac{\Delta \nu}{\nu}=-(\theta \beta)^{2}$, where $\theta$ is the angle between lasers and ion beam. With the alignment procedures described above the residual angles of $\phi< \pm 40 \mu \mathrm{rad}$ and $\theta< \pm 370 \mu \mathrm{rad}$ and frequency uncertainties of $\Delta \nu= \pm 30 \mathrm{kHz}$ and $\Delta \nu= \pm 285 \mathrm{kHz}$ were achieved. A summary of all errors contributing to the present experiment is given in table (1).

The largest uncertainty is related to the line width of the $\Lambda$ resonance of $\Delta \nu_{F W H M} \leq 60$ $\mathrm{MHz}$ which exceeds almost twice the line width due to saturation, time of flight and modulation broadening.

Line broadening by intra beam scattering or space charge effects is ruled out by observing a constant line width for a change in the ion beam intensity by more than a factor of 3. The observed line width seems to be mainly due to phasefront perturbations in the $\mathrm{TEM}_{00}$ laser beams interacting with fast ions in a broad velocity distribution. A numerical solution of the optical Bloch equations for a model ion beam interacting with the $\mathrm{TEM}_{00}$ aligned laser beams shows indeed a line broadening for typical TSR parameters. Moreover an interferometric analysis of the laser beams resulted in the observation of additional perturbations due to the weak optical quality of one of the TSR entrance windows. Phaseshifts of $6 \pi$ over a laser beam diameter of $11 \mathrm{~mm}$ have been deduced from 


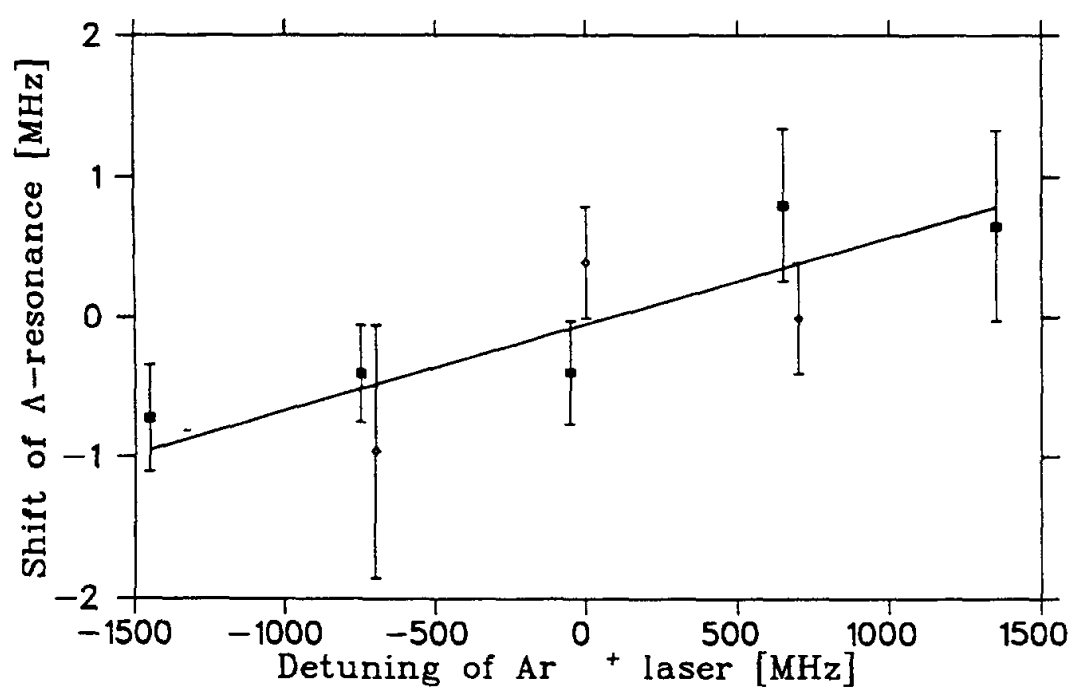

Figure 6: AC Stark shift of the $\Lambda$ resonance when detuned from the center of the Doppler profile of the velocity distribution. The Doppler tuning has been controlled by the cathode potential of the electron cooler. A setting accuracy of $\Delta \nu= \pm 1 \mathrm{MHz}$ is deduced.

\begin{tabular}{|l|c|}
\hline & Accuracy [MHz] \\
\hline \hline interpolation error for frequency offset & $<0.85$ \\
\hline shift by angle $\theta$ ion/laser & $<0.285$ \\
\hline shift by residual angle $\phi$ between lasers & $<0.03$ \\
\hline $\begin{array}{l}\text { AC Stark shift by offset in electron } \\
\text { cooler velocity }\end{array}$ & $<1.0$ \\
\hline shift due to photon recoil & $<0.7$ \\
\hline shifts due to wave front distortion & $<2.7$ \\
\hline error in lifetime correction & $<0.5$ \\
\hline fit error & $<0.08$ \\
\hline \hline total & $<3.1$ \\
\hline
\end{tabular}

Table 1: Systematic errors on the $1 \sigma$ level for the experimental determination of the position of the $\Lambda$ resonance. Details are given in the text. 
the observed fringes for the corresponding aberrations. Because these phase distortions are not symmetric with respect to the laser axis, a corresponding frequency uncertainty of $\Delta \nu= \pm 2.7 \mathrm{MHz}$ has to be taken into account, assuming the estimated accuracy of the angular beam alignment. 


\begin{tabular}{|l||c||c|}
\hline & & $\mathrm{MHz}$ \\
\hline \hline Transition frequencies & ${ }^{3} \mathrm{~S}_{1} \mathrm{~F}=\frac{5}{2} \rightarrow{ }^{3} \mathrm{P}_{2} \mathrm{~F}^{\prime}=\frac{7}{2}$ & $\nu_{5 / 2 \rightarrow 7 / 2}=546466919.9(1.7)$ \\
in ${ }^{7} L^{+}$at rest & ${ }^{2} P_{2} F^{\prime}=\frac{5}{2} \rightarrow{ }^{2} P_{2} F^{\prime}=\frac{7}{2}$ & $\nu=11775.8(2)$ \\
& ${ }^{3} \mathrm{~S}_{1} \mathrm{~F}=\frac{5}{2} \rightarrow{ }^{3} \mathrm{P}_{2} \mathrm{~F}^{\prime}=\frac{5}{2}$ & $\nu_{5 / 2 \rightarrow 5 / 2}=546455144.1(1.7)$ \\
& ${ }^{3} S_{1} F=\frac{3}{2} \rightarrow{ }^{3} S_{1} F=\frac{5}{2}$ & $\nu=19817.673(13)$ \\
& ${ }^{3} \mathrm{~S}_{1} \mathrm{~F}=\frac{3}{2} \rightarrow{ }^{3} \mathrm{P}_{2} \mathrm{~F}^{\prime}=\frac{5}{2}$ & $\nu_{3 / 2 \rightarrow 5 / 2}=546474961.8(1.7)$ \\
\hline $\mathrm{Ar}^{+}$laser stabilized on & $\mathrm{a}_{3}$ line of $\mathrm{P}(13) 43-0$ & $\nu_{A r}=582490603.370(130)$ \\
\hline$\rightarrow$ Special relativity & $\nu_{5 / 2 \rightarrow 5 / 2} \cdot \nu_{3 / 2 \rightarrow 5 / 2} / \nu_{A T}$ & $\nu_{S R T}=512667590.2(3.2)$ \\
\hline Measured frequency & & $\nu_{E x p}=512667592.4(3.1)$ \\
\hline \hline Difference & & $\Delta \nu=2.2(4.5)$ \\
\hline
\end{tabular}

Table 2: Frequencies involved in the test experiment. The absolute transition frequency of the ${ }^{3} S_{1} F=\frac{5}{2} \rightarrow{ }^{3} P_{2} F^{\prime}=\frac{7}{2}$ is taken from [15], the ${ }^{7} \mathrm{Li}^{+}$HFS splitting is taken from [16] and the frequency of the stabilized $\mathrm{Ar}^{+}$laser is taken from [21]. The measured frequency is the sum of the frequency of the $R(99) 15-1 \mathrm{i}$-component in ${ }^{127} I_{2}[22]$ and the measured frequency difference between this line and the position of the $\Lambda$ resonance. All errors correspond to the $1 \sigma$ level.

\section{Results and discussion}

Relative to the i-component of the ${ }^{127} I_{2} \mathrm{R}(99) 15-1$ resonance line the line positions of the $\Lambda$ resonance in ten corrected sum spectra have been used to determine a weighted mean value. The result together with its $1 \sigma$ error resulting from table (1) is given as the measured frequency

$$
\nu_{E x p}=512667592.4(3.1) M H z
$$

in table (2). The product of the frequencies of the $F=\frac{3}{2} \rightarrow F^{\prime}=\frac{5}{2}$ and $F=\frac{5}{2} \rightarrow F^{\prime}=\frac{5}{2}$ transitions in ${ }^{7} \mathrm{Li}^{+}$at rest divided by the frequency of the $\mathrm{Ar}^{+}$laser locked on the $\mathrm{a}_{3}$ component of the $\mathrm{P}(13) 43-0{ }^{127} I_{2}$ line is quoted as the special relativity value

$$
\nu_{S R T}=512667590.2(3.2) M H z .
$$

The frequency difference $\Delta \nu=2.2 \pm 4.5 \mathrm{MHz}$ compatible with zero is confirming the validity of special relativity, and using equation (1) a limit for the test theory value $\delta \alpha<1.1 \cdot 10^{-6}$ is deduced for a velocity $\beta=0.064$. For similar or higher velocities this result supersedes our previous result a factor of 14 and is at least two orders of magnitude more accurate than a Doppler shift experiment on the hydrogen $1 \mathrm{~s} \rightarrow 5 \mathrm{p}$ line at $\beta=0,84$ [24]. Moreover it slightly improves the Neon experiments performed at $\beta=0.0036$ [13] [12] for testing frequency shift and frequency modulation with sidereal time, assuming an "ether" frame moving at $\mathrm{v}^{*}=350 \mathrm{~km} / \mathrm{s}$. A sidereal modulation test by Mößbauer 
experiments was announced to give $\delta \alpha<10^{-7}[10]$.

Although the expansion of test theories to higher orders in $\beta^{2}$ given in [8] is adapted to low velocity experiments, the higher order components are mainly limited by the experimental results at high velocities. Following reference [8] the fourth order contribution to the test theory is given by $\frac{\Delta \nu}{\nu}=2 \cdot \delta \alpha_{2} \beta^{4}$. With the limit of $\delta \alpha$ from the low velocity tests, the present experiment results in $\delta \alpha_{2}<2.7 \cdot 10^{-4}$. The high velocity hydrogen experiment [24] can be analyzed in terms of $\delta \alpha_{2}$, and assuming $\delta \alpha<10^{-7}$ taken from [10], leads to $\delta \alpha_{2}<1.1 \cdot 10^{-4}$, which is still more accurate than the present experiment.

In the (g-2) experiments with relativistic leptons in a magnetic field $B$ the difference of spin precession $\omega_{s}$ and cyclotron frequency $\omega_{c}$ is measured as

$$
\omega_{s}-\omega_{c}=\left(\frac{g}{2}-\frac{\gamma}{\tilde{\gamma}}\right) \frac{e}{m c} B
$$

where the time dilatation factor $\gamma$ is compared with the factor $\bar{\gamma}$ of special relativity applied to accelerated systems [25]. The experiment at the CERN muon storage ring [26] quotes at $\beta=0.9994$ and $\dot{\beta}=3 \cdot 10^{10} \mathrm{~s}^{-1}$ a invariance limit in accelerated systems of $\frac{\delta \gamma}{\tilde{\gamma}}<2.7 \cdot 10^{-4}$.

In the combined analysis of precise electron ( $\mathrm{g}-2)$ experiments at $\beta=0.57$ and at rest discussed in [25] an invariance limit of $\frac{\delta \gamma}{\tilde{\gamma}}<5.3 \cdot 10^{-9}$ at $\dot{\beta}=4 \cdot 10^{9} \mathrm{~s}^{-1}$ is given. These experiments are independent of $\beta$, except for experimental corrections and are therefore not suited for a test of $\gamma^{2}\left(1-\beta^{2}\right)=1$.

The accuracy potential of our Doppler shift experiment can be reached by more accurate values for the ${ }^{7} \mathrm{Li}^{+}$transition frequency at rest relative to the calibrated $\mathrm{R}(85) 26-0$ w-component in ${ }^{127} I_{2}[22]$ and by the elimination of phasefront perturbation in the $\Lambda$ resonance interaction zone. Considering moreover a precise velocity control by simultaneous laser cooling of the ${ }^{7} \mathrm{Li}^{+}$beam the final accuracy should be determined by the angular setting of laser and ion beam, which has been quoted as $\Delta \nu<285 \mathrm{kHz}$ in table (1). Compared to the $1 \sigma$ error in the present experiment of $4.5 \mathrm{MHz}$ the ultimate limit imposed by imperfections in beam alignment and signal distortions by time of fight effects in the interaction region should allow a gain by a factor of 15 in testing the special theory of relativity, resulting in a further improvement in $\delta \alpha$ by the same amount.

The limits of the ${ }^{7} \mathrm{Li}^{+}$experiment may be reduced considerably in a two-photon experiment on neutral hydrogen prepared from an electron cooled particle beam in a storage ring. Proton beams can be stored and cooled up to $\beta \sim 0.2$ at the TSR ring and to $\beta \sim 0.72$ at the ESR ring at GSI/Darmstadt, and a weak neutralized beam by radiative 
electron capture in the 1s state of hydrogen could be extracted from the ring. The highly monochromatic low emittance atomic beam can be excited to a Rydberg state by twophoton resonance with counter-propagating and parallel laser beams. By careful matching the atomic beam emittance and the laser beams in a long interaction section $(L<50 \mathrm{~m})$ precise angular alignment $(\theta<20 \mu \mathrm{rad})$ and small signal broadening could be realized. For these conditions a state of the art frequency measurement at the ESR of the $1 \mathrm{~s} \rightarrow$ $3 p$ resonance driven with an antiparallel aligned frequency doubled $\mathrm{Ar}^{+}$laser running at $514 \mathrm{~nm}$ and the $3 \mathrm{p} \rightarrow 8 \mathrm{~d}$ resonance driven with a parallel aligned frequency doubled Ti-Sapphire laser running at $761 \mathrm{~nm}$ would result in a resolution of $\frac{\Delta \nu}{\nu}<2.5 \cdot 10^{-10}$. A corresponding value $\delta \alpha<5 \cdot 10^{-10}$ should be reachable, testing the special theory of relativity over three orders of magnitude more accurately than in the present experiment. Acknowledgement: The authors thank J.Helmcke and his division at the PTB Braunschweig for their support in laser stabilization. This work is supported by Bundesministerium für Forschung und Technologie and GSI.

\section{References}

[1] A.A.Michelson and E.W.Morley, Am.J.Sci. 34,333 (1887)

[2] R.J. Kennedy and E.M. Thorndike, Phys. Rev. 42,400 (1932)

[3] A. Brillet and J.L. Hall, Phys.Rev.Lett. 42, 549 (1979)

D. Hils and J.L. Hall, Phys.Rev.Lett. 64, 1697 (1990)

[4] B.J. Venema, P.K. Majumder, S.K. Lamoreaux, B.R.Heckel, E.N.Fortson, Phys.Rev.Lett. 68, 135 (1992)

M.P. Haugan, C.M. Will, Phys. Today 40 (5), 69 (1987)

[5] H.E.Ives and G.R.Stilwell, J.Opt.Soc. Am. 28,215 (1938)

[6] R.Mansouri and R.Sexl, Gen.Rel. and Grav. 8(7), 497 (1977)

R.Mansouri und R.Sexl, Gen.Rel. and Grav. 8(7), 515 (1977)

R.Mansouri und R.Sexl, Gen.Rel. and Grav. 8(7), 809 (1977)

[7] G.F. Smoot, M.V.Gorenstein, Phys.Rev.Lett. 39, 898 (1977)

[8] C.M.Will, Phys.Rev. D 45, 403 (1992) 
[9] M.Kretzschmar, Z Phys. A 342, 463 (1992)

[10] G.R. Isaac, Phys.Bull. 21, 255 (1970)

[11] M.Kaivola, O.Poulsen, E.Riis, S.A.Lee, Phys. Rev. Lett. 54, 255 (1985)

[12] R.W.McGowan, D. M.Giltner, S. J.Sternberg, S. A. Lee, Phys. Rev. Lett. 70, 251 (1993)

[13] E.Riis, L.U.A. Andersen, N.Bjerre, O.Poulsen, S.A.Lee, J.L.Hall, Phys. Rev. Lett. 60, 81 (1988)

[14] R.Klein, R.Grieser, I.Hoog, G.Huber, I.Klaft, P.Merz, T.Kühl, S.Schröder, M.Grieser, D.Habs, W.Petrich, D. Schwalm, Z Phys. A 342, 455 (1992)

[15] E.Riis, H.G.Berry, O.Poulsen, S.A.Lee, S.Y.Tang, Phys. Rev. A 33, 3023 (1986)

[16] J.Kowalski, R.Neumann, S.Noethe, K.Scheffzek, H.Suhr, G. zu Putlitz, Hyperf. Interact. 15/16, 159 (1983) H.Rong, S.Grafström, J.Kowalski, G.zu Putlitz, W.Jastrzebski, R.Neumann, Z.Physik.D 25, 337 (1993)

[17] P.Baumann, M.Blum, A.Friedrich, C.Geyer, M.Grieser, B.Holzer, E.Jaeschke, D.Krämer, R.Mayer, C.Martin, K.Matl, W.Ott, B.Povh, R.Repnow, M.Steck, E.Steffens, Nucl.Instrum.Methods a 268,531 (1988) D.Habs , Nuclear Physics News 4, 17 (1991)

[18] G.I.Budker, N.S.Dikanskii, V.I.Kudelainen, I.N.Meshkov, V.V.Parkhomchuk, D.V. Pestrikov, A.N. Skrinski, B.N.Sukhina, Part.Accel.7, 197 (1976)

H.Poth, W.Schwab, B.Seligmann, M.Wörtge, A.Wolf, S.Baird, M.Chanel, H.Haseroth, C.E.Hill, R.Ley, D.Manhlunki, D.Tranquille, J.L.Vallet, P.F.Dittner, Z Phys.A-Atomic Nuclei 332, 171 (1989) E.Jaeschke et al., Proc.European Particle Accelerator Conf., Rome 1988. Tazzari (ed.) p.365, Singapore: World Scientific (1989) 
[19] S.Schröder, R.Klein, N.Boos, M.Gerhard, R.Grieser, G.Huber, A.Karafillidis, M.Krieg, N.Schmitt, T.Kühl, R.Neumann, V.Balikyn, M.Grieser, D.Habs, E.Jaeschke, D.Kraemer, M.Kristensen, M.Music, W.Petrich, D.Schwalm, P.Sigray, M.Steck, B.Wanner, A.Wolf, Phys. Rev. Lett. 64, 2901 (1990)

G.Huber, S.Schröder, R.Klein, N.Boos, R.Grieser, I.Hoog, M.Krieg, P.Merz, T.Kühl, R.Neumann, V.Balikyn, M.Grieser, D.Habs, E.Jaeschke, W.Petrich, D.Schwalm, M.Steck, B.Wanner, A.Wolf, Atomic Physics 12, New York:AIP 1991

[20] F.Albrecht, Thesis , Heidelberg 1993

[21] Documents concerning the New Definition of the Metre, Metrologia 19, 163, (1984) Comité International des Poids et Mesures (CIPM), $81^{e}$ session, 1992, Recommendation 3, S.144

[22] R.Grieser, S.Dickopf, G.Huber, R.Klein, P.Merz, G.Bönsch, A.Niclolaus, H.Schnatz, to be published

[23] U.Brand, PTB-Opt-34, Braunschweig, 1991

[24] D.W. MacArthur, K.B.Butterfield, D.A.Clark, J.B.Donahue, P.A.M.Gram, H.C.Bryant, C.J.Harvey, W.W.Smith, G.Comtet, Phys. Rev. Lett. 56, 282 (1986)

[25] D.Newman, G.W.Ford, A.Rich, E.Sweetman, Phys. Rev. Lett. 40, 1355 (1978)

[26] J. Bailey, K.Borer, F.Combley, H.Drumm, F.J.M.Farley, J.H.Field, W.Flegel, P.M. Hattersley, F.Krienen, F.Lange, E.Picasso, W.Von Rüden, Phys. Lett. 68B (2), 191 (1977) 